\title{
On the Endomorphisms of Weyl Modules over Affine Kac-Moody Algebras at the Critical Level
}

\author{
BORIS FEIGIN ${ }^{1}$, EDWARD FRENKEL ${ }^{2}$ and LEONID RYBNIKOV ${ }^{3}$ \\ ${ }^{1}$ Landau Institute for Theoretical Physics, Kosygina St 2, 117940 Moscow, Russia. \\ e-mail: bfeigin@gmail.com \\ ${ }^{2}$ Department of Mathematics, University of California, Berkeley, CA 94720, USA. \\ e-mail:frenkel@Math.Berkeley.edu \\ ${ }^{3}$ Institute for Theoretical and Experimental Physics, B. Cheremushkinskaya 25, \\ 117259 Moscow, Russia.e-mail: leo.rybnikov@gmail.com
}

Received: 7 December 2008 / Accepted: 14 February 2009

Published online: 27 February 2009 - (C) The Author(s) 2009. This article is published with open access at Springerlink.com

\begin{abstract}
We present an independent short proof of the recently established result that the algebra of endomorphisms of a Weyl module of critical level is isomorphic to the algebra of functions on the space of monodromy-free opers on the disc with regular singularity and residue determined by the highest weight of the Weyl module. We derive this from our results about the shift of argument subalgebras.
\end{abstract}

Mathematics Subject Classification (2000). 81R10.

Keywords. affine Kac-Moody algebra at critical level, Weyl module, oper, monodromy.

\section{Formulation of the Main Result}

\subsection{WEYL MODULES AT THE CRITICAL LEVEL}

Let $\mathfrak{g}$ be a simple Lie algebra, and $\widehat{\mathfrak{g}}$ be the corresponding affine Kac-Moody algebra. The Lie algebra $\widehat{\mathfrak{g}}$ is a central extension of the formal loop algebra $\mathfrak{g}((t))$ by one-dimensional center with generator 1 . The commutation relations are as follows:

$$
\left[g_{1} \otimes x(t), g_{2} \otimes y(t)\right]=\left[g_{1}, g_{2}\right] \otimes x(t) y(t)+\kappa_{c}\left(g_{1}, g_{2}\right) \operatorname{Res}_{t=0} x(t) \mathrm{d} y(t) \cdot \mathbf{1},
$$

B. Feigin was supported by the grants RFBR 08-01-00720, RFBR 05-01-02934 and NSh-6358.2006.2.

E. Frenkel was supported by DARPA and AFOSR through the grant FA9550-07-1-0543.

L. Rybnikov was supported by RFBR 05-01-02805-CNRSL-a, RFBR 07-01-92214-CNRSL-a, NSF grant DMS-0635607 and Deligne 2004 Balzan prize in mathematics. L. Rybnikov gratefully acknowledges the Institute for Advanced Study for providing warm hospitality and excellent working conditions. 
where $\kappa_{c}$ is the invariant inner product on $\mathfrak{g}$ defined by the formula

$$
\kappa_{c}\left(g_{1}, g_{2}\right)=-\frac{1}{2} \operatorname{Tr}_{\mathfrak{g}} \operatorname{ad}\left(g_{1}\right) \operatorname{ad}\left(g_{2}\right) .
$$

Set $\widehat{\mathfrak{g}}_{+}=\mathfrak{g}[[t]] \subset \widehat{\mathfrak{g}}$ and $\widehat{\mathfrak{g}}_{-}=t^{-1} \mathfrak{g}\left[t^{-1}\right] \subset \widehat{\mathfrak{g}}$.

Define the completion $\widetilde{U}(\widehat{\mathfrak{g}})$ of $U(\widehat{\mathfrak{g}})$ as the inverse limit of $U(\widehat{\mathfrak{g}}) / U(\widehat{\mathfrak{g}})\left(t^{n} \mathfrak{g}[[t]]\right)$, $n>0$. The action of $\widetilde{U}(\widehat{\mathfrak{g}})$ is well-defined on the category of discrete $\widehat{\mathfrak{g}}$-modules, i.e., those in which every vector is annihilated by $t^{n} \mathfrak{g}[[t]]$ for some $n>0$. We set

$$
\widetilde{U}_{\kappa_{c}}(\widehat{\mathfrak{g}})=\widetilde{U}(\widehat{\mathfrak{g}}) /(\mathbf{1}-1) .
$$

This algebra acts on discrete $\widehat{\mathfrak{g}}$-modules of critical level (i.e., $\widehat{\mathfrak{g}}$-modules on which the element $K$ acts as unity).

For a dominant integral weight $\lambda$ of $\mathfrak{g}$, let

$$
\pi_{\lambda}: \mathfrak{g} \rightarrow \operatorname{End}_{\mathbb{C}} V_{\lambda}
$$

be the finite-dimensional irreducible representation of $\mathfrak{g}$ with the highest weight $\lambda$. One can naturally extend this representation to $\widehat{\mathfrak{g}}_{+}=\mathfrak{g}[[t]]$ by using the composition with the natural map $\mathfrak{g}[[t]] \rightarrow \mathfrak{g}$ corresponding to evaluation at $t=0$. The Weyl module at the critical level with the highest weight $\lambda$ is by definition the induced module

$$
\mathbb{V}_{\lambda}:=\operatorname{Ind}_{\widehat{\mathfrak{g}}_{+} \oplus \mathbb{C} 1}^{\widehat{\widehat{a}}} V_{\lambda},
$$

where 1 acts on $V_{\lambda}$ as the identity.

\subsection{ACTION OF THE CENTER AND MONODROMY-FREE OPERS}

Consider the Langlands dual Lie algebra ${ }^{L} \mathfrak{g}$ whose Cartan matrix is the transpose of the Cartan matrix of $\mathfrak{g}$. Denote by ${ }^{L} G$ the group of inner automorphisms of $L_{\mathfrak{g}}$. In $[2,8]$ the center $Z(\widehat{\mathfrak{g}})$ of the completed enveloping algebra $\widetilde{U}_{\kappa_{c}}(\widehat{\mathfrak{g}})$ at the critical level was identified with the algebra of polynomial functions on the space $\mathrm{Op}_{L_{G}}\left(D^{\times}\right)$of ${ }^{L} G$-opers on the $\operatorname{disc} D=\operatorname{Spec} \mathbb{C}((t))$.

Let us recall the notion of opers which was introduced in [1]. Fix a Cartan decomposition

$$
{ }^{L} \mathfrak{g}={ }^{L} \mathfrak{n} \oplus{ }^{L} \mathfrak{h} \oplus{ }^{L} \mathfrak{n}_{-} .
$$

The Cartan subalgebra ${ }^{L} \mathfrak{h}$ is canonically identified with $\mathfrak{h}^{*}$. We denote by $\Pi^{\vee}$ the set of simple roots of ${ }^{L} \mathfrak{g}$ (which is the set of simple coroots of $\mathfrak{g}$ ). Set

$$
p_{-1}=\sum_{\alpha^{\vee} \in \Pi^{\vee}} e_{-\alpha^{\vee}} \in{ }^{L} \mathfrak{g},
$$

where the $e_{-\alpha^{\vee}}$ are non-zero generators of the $-\alpha^{\vee}$-root subspaces in ${ }^{L} \mathfrak{n}_{-}$. 
The space $\mathrm{Op}_{L}\left(D^{\times}\right)$of ${ }^{L} G$-opers is the quotient of the space of connections on the trivial ${ }^{L} G$-bundle on $D^{\times}$of the form

$$
d+\left(p_{-1}+\mathbf{v}(z)\right) d z, \quad \mathbf{v}(z) \in^{L} \mathfrak{b}((z))
$$

by the action of the group ${ }^{L} N((z))$.

Consider the action of the center $Z(\widehat{\mathfrak{g}})$ on $\mathbb{V}_{\lambda}$. Since $Z(\widehat{\mathfrak{g}})=\mathrm{Fun}\left(\mathrm{Op}_{L_{G}}\left(D^{\times}\right)\right)$, the support of $\mathbb{V}_{\lambda}$ as a $Z(\widehat{\mathfrak{g}})$-module is a closed subset in the space of opers $\mathrm{Op}_{L_{G}}\left(D^{\times}\right)$.

In [6], Sect. 2.9, a closed subspace $\mathrm{Op}_{L_{G}}^{\lambda, \text { reg }} \subset \mathrm{Op}_{L_{G}}\left(D^{\times}\right)$of monodromy-free opers with regular singularity and residue determined by $\lambda$ was defined (this definition is reviewed in [9, Sect. 9.2.3], and in [4, Sect. 4.4]).

The following assertion was proved in [7, Lemma 1.7].

PROPOSITION 1. The support of $\mathbb{V}_{\lambda}$ is contained in the subspace $\mathrm{Op}_{L_{G}}^{\lambda, \text { reg }} \subset$ $\mathrm{Op}_{L_{G}}\left(D^{\times}\right)$.

Furthermore, in [5] the following theorem was proved which completely describes the algebra of endomorphisms of the Weyl module $\mathbb{V}_{\lambda}$ :

THEOREM 1. There is a commutative diagram

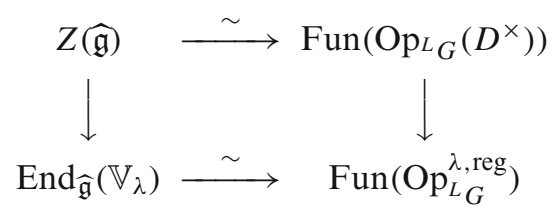

The proof of this theorem given in [5] used non-trivial results about the semiinfinite cohomology of $\mathbb{V}_{\lambda}$. The goal of this paper is to give an alternative proof of this theorem, in which we will not use semi-infinite cohomology, but will rely instead the results about the shift of argument subalgebra from [3] and on Proposition 1 .

\subsection{IDEA OF THE PROOF}

The loop rotation operator $-t \partial_{t}$ acting on $\widehat{\mathfrak{g}}$ defines a $\mathbb{Z}$-grading on $Z(\widehat{\mathfrak{g}})$ and on Fun $\left(\mathrm{Op}_{L_{G}}\left(D^{\times}\right)\right)$, and the isomorphism of [2] preserves these gradings.

According to Proposition 1, the action of $Z(\widehat{\mathfrak{g}})$ on $\mathbb{V}_{\lambda}$ factors through the algebra $\operatorname{Fun}\left(\mathrm{Op}_{L_{G}}^{\lambda, \text { reg }}\right)$. Hence it is sufficient to prove that the map $Z(\widehat{\mathfrak{g}}) \rightarrow \operatorname{End}_{\mathfrak{\mathfrak { g }}}\left(\mathbb{V}_{\lambda}\right)$ is surjective, and that the character of $\operatorname{Fun}\left(\mathrm{Op}_{L_{G}}^{\lambda, \text { reg }}\right)$ (understood as the formal power series in a variable $q$ whose $q^{n}$ coefficient is the dimension of the degree $n$ subspace) is not greater, term by term, than that of $\operatorname{End}_{\widehat{\mathfrak{g}}}\left(\mathbb{V}_{\lambda}\right)$. 
The character of Fun $\left(\mathrm{Op}_{L_{G}}^{\lambda, \text { reg }}\right)$ was computed in [5], Sect. 5.1, and is given by the formula

$$
\frac{\prod_{\alpha>0}\left(1-q^{\left\langle\alpha^{\vee}, \lambda+\rho\right\rangle}\right)}{\prod_{k=1}^{\infty}\left(1-q^{k}\right)^{\mathrm{rkg}}} .
$$

Here is a brief derivation of this formula. Recall that the algebra Fun $\left(\mathrm{Op}_{L_{G}}^{\lambda \text {,reg }}\right)$ is the quotient of the algebra $\operatorname{Fun}\left(\mathrm{Op}_{L} G\left(D^{\times}\right)_{\lambda}^{R S}\right)$, where $\mathrm{Op}_{L}^{\lambda}{ }_{G}$, RS is the space of opers with regular singularity and residue $\lambda$. The latter is a free polynomial algebra with homogeneous generators $P_{i}^{(k)}$, where $i=1, \ldots, \mathrm{rkg}, k \geq 0$, whose degree is equal to $k$. The subset $\mathrm{Op}_{L}^{\lambda,}{ }_{G}^{\lambda,} \subset \mathrm{Op}_{L}^{\lambda,}{ }_{G}^{\lambda S}$ is defined by a regular sequence of homogeneous relations, enumerated by positive roots $\alpha$ of $\mathfrak{g}$, of the degree $\left\langle\alpha^{\vee}, \lambda+\rho\right\rangle$ (see the above references $[4,6,9]$ for details). This gives us formula (3).

In order to estimate the character of $\operatorname{End}_{\widehat{\mathfrak{g}}}\left(\mathbb{V}_{\lambda}\right)$, we pass from $\operatorname{End}_{\widehat{\mathfrak{g}}}\left(\mathbb{V}_{\lambda}\right)$ to its associated graded algebra with respect to the PBW filtration. The crucial points in our proof are the result of [3] that $V_{\lambda}$ is a cyclic module over the nilpotent shift of argument subalgebra $\mathcal{A}_{f}$ (which turns out to be closely related to the associated graded algebra $\left.\operatorname{gr} \operatorname{End}_{\widehat{\mathfrak{g}}}\left(\mathbb{V}_{\lambda}\right)\right)$ and the computation of invariants from [9]. Using these results, we obtain the desired lower bound for the character of $\operatorname{gr} \operatorname{End}_{\widehat{\mathfrak{g}}}\left(\mathbb{V}_{\lambda}\right)$.

\section{Proof of the Theorem}

\subsection{SHIFT OF ARGUMENT SUBALGEBRAS}

To any $\mu \in \mathfrak{g}^{*}$ one can assign a commutative subalgebra $\mathcal{A}_{\mu} \subset U(\mathfrak{g})$ called the quantum shift of argument subalgebra. This algebra comes from the center $Z(\widehat{\mathfrak{g}})$ in the following way. Let $\pi: \widehat{\mathfrak{g}}_{+} \rightarrow \mathfrak{g}$ be the homomorphism of evaluation at $t=0$. Consider the following quantum Hamiltonian reduction algebra

$$
\left(U(\mathfrak{g}) \otimes \widetilde{U}_{\kappa_{c}}(\widehat{\mathfrak{g}}) / U(\mathfrak{g}) \otimes \widetilde{U}_{\kappa_{c}}(\widehat{\mathfrak{g}})\left(\widehat{\mathfrak{g}}_{+}-\pi\left(\widehat{\mathfrak{g}}_{+}\right)\right)\right)^{\widehat{\mathfrak{g}}_{+}} .
$$

Here $U(\mathfrak{g}) \otimes \widetilde{U}_{\kappa_{c}}(\widehat{\mathfrak{g}})\left(\widehat{\mathfrak{g}}_{+}-\pi\left(\widehat{\mathfrak{g}}_{+}\right)\right)$is the left ideal generated by $x-\pi(x)$ for all $x \in$ $\widehat{\mathfrak{g}}_{+}$. The center $Z(\widehat{\mathfrak{g}})$ of $\widetilde{U}_{\kappa_{c}}(\widehat{\mathfrak{g}})$ naturally maps to this quotient. Each element of the above quotient has a unique representative in $U(\mathfrak{g}) \otimes U\left(\widehat{\mathfrak{g}}_{-}\right) \subset U(\mathfrak{g}) \otimes \widetilde{U}_{\kappa_{c}}(\widehat{\mathfrak{g}})$. Thus we obtain a homomorphism $Z(\widehat{\mathfrak{g}}) \rightarrow U(\mathfrak{g}) \otimes U\left(\widehat{\mathfrak{g}}_{-}\right)$.

The element $\mu \in \mathfrak{g}^{*}$ defines a character $\mu_{-}: \widehat{\mathfrak{g}}_{-} \rightarrow \mathbb{C}$ by $\mu_{-}\left(x t^{-1}\right)=\langle\mu, x\rangle$ and $\mu_{-}\left(x t^{-k}\right)=0$ for $k>1$. This gives us a homomorphism

$$
\operatorname{id} \otimes \mu_{-}: U(\mathfrak{g}) \otimes U\left(\widehat{\mathfrak{g}}_{-}\right) \rightarrow U(\mathfrak{g}) .
$$

Thus, we have a homomorphism $Z(\widehat{\mathfrak{g}}) \rightarrow U(\mathfrak{g})$ depending on $\mu \in \mathfrak{g}^{*}$. The subalgebra $\mathcal{A}_{\mu} \subset U(\mathfrak{g})$ is, by definition, the image of this homomorphism (see $[4,11]$ for more details).

Let $\mathfrak{Z}$ be the image of the center $Z(\widehat{\mathfrak{g}})$ in $U(\mathfrak{g}) \otimes U\left(\widehat{\mathfrak{g}}_{-}\right)$. It follows from [4, Theorem 5.6(1) and Lemma 5.5], that $\mathfrak{Z}$ is a free commutative algebra generated by the homogeneous (with respect to the grading defined by the loop-rotation 
operator $-t \partial_{t}$ ) elements $P_{i}^{(k)}$ of degree $k, i=1, \ldots, \ell=\mathrm{rk} \mathfrak{g}, k=0,1, \ldots$, such that $P_{i}^{(0)} \in U(\mathfrak{g}) \otimes 1$ are the generators of the center of $U(\mathfrak{g})$. The degree of $P_{i}^{(k)}$ with respect to the PBW filtration is $d_{i}+1$, where $d_{1}, \ldots, d_{\ell}$ are the exponents of $\mathfrak{g}$. From the description of these elements given in the above reference it is easy to see that the associated graded of $\mathfrak{Z}$ with respect to the PBW filtration on the second factor of $U(\mathfrak{g}) \otimes U\left(\widehat{\mathfrak{g}}_{-}\right)$is freely generated by elements $\overline{P_{i}^{(k)}}, i=1, \ldots, \mathrm{rkg}, k \geq 0$, such that $\overline{P_{i}^{(k)}} \in U(\mathfrak{g}) \otimes S\left(\widehat{\mathfrak{g}}_{-}\right)$for $i=1, \ldots, \mathrm{rk} \mathfrak{g}, k=0,1, \ldots, d_{i}$, where the $d_{i}$ are the exponents of $\mathfrak{g}$, and $\overline{P_{i}^{(k)}} \in 1 \otimes S\left(\widehat{\mathfrak{g}}_{-}\right)$for $i=1, \ldots$, rk $\mathfrak{g}, k>d_{i}$.

LEMMA 1. (see also [4, Lemma 3.13]) For regular $\mu$ the images of $\mathfrak{Z} \subset U(\mathfrak{g}) \otimes$ $U\left(\widehat{\mathfrak{g}}_{-}\right)$and $\operatorname{gr} \mathfrak{Z} \subset U(\mathfrak{g}) \otimes S\left(\widehat{\mathfrak{g}}_{-}\right)$under $\mathrm{id} \otimes \mu_{-}$and $\mathrm{id} \otimes \operatorname{gr} \mu_{-}$, respectively, coincide and are equal to the same commutative subalgebra $\mathcal{A}_{\mu} \subset U(\mathfrak{g})$.

Proof. According to [11, Theorem 1] and [4, Theorem 5.8], for any regular $\mu \in \mathfrak{g}^{*}$ the subalgebra $\mathcal{A}_{\mu} \subset U(\mathfrak{g})$ is freely generated by the images of $P_{i}^{(k)}$ with $i=1, \ldots$, rk $\mathfrak{g}, k=0,1, \ldots, d_{i}$, where the $d_{i}$ are the exponents of $\mathfrak{g}$. Since the elements $P_{i}^{(k)}$ are homogeneous with respect to the loop rotation operator, the image of $P_{i}^{(k)}$ in $U(\mathfrak{g}) \otimes S\left(\mathfrak{g} t^{-1}\right)=U(\mathfrak{g}) \otimes U\left(t^{-1} \mathfrak{g}\left[t^{-1}\right] /\left(1 \otimes t^{-2} \mathfrak{g}\left[t^{-1}\right]\right)\right.$ is homogeneous with respect to the grading on the second factor, and hence coincides with the image of $\overline{P_{i}^{(k)}}$ in $U(\mathfrak{g}) \otimes S\left(\mathfrak{g} t^{-1}\right)=U(\mathfrak{g}) \otimes S\left(t^{-1} \mathfrak{g}\left[t^{-1}\right] /\left(1 \otimes t^{-2} \mathfrak{g}\left[t^{-1}\right]\right)\right.$. Since the homomorphism id $\otimes \mu_{-}$factors through $U(\mathfrak{g}) \otimes S\left(\mathfrak{g} t^{-1}\right)$, we have

$$
\mathrm{id} \otimes \mu_{-}\left(P_{i}^{(k)}\right)=\mathrm{id} \otimes \operatorname{gr} \mu_{-}\left(\overline{P_{i}^{(k)}}\right) .
$$

Therefore the images of $\overline{P_{i}^{(k)}} \in U(\mathfrak{g}) \otimes S\left(\widehat{\mathfrak{g}}_{-}\right)$with $k=0,1, \ldots, d_{i}$ under the map id $\otimes \operatorname{gr} \mu_{-}$generate the same commutative subalgebra $\mathcal{A}_{\mu} \subset U(\mathfrak{g})$.

We remark that a certain limit of $\mathcal{A}_{\mu}$ in the case when $\mathfrak{g}=\mathfrak{s l}_{n}$ may be identified with the Gelfand-Zetlin algebra (see [11]). Hence the algebra $\mathcal{A}_{\mu}$ may be thought of as a generalization of the Gelfand-Zetlin algebra to an arbitrary simple Lie algebra.

An important special case is when $\mu \in \mathfrak{g}^{*} \simeq \mathfrak{g}$ is a regular nilpotent element. Since all of these elements belong to a single coadjoint orbit, it is sufficient to consider one particular representative. Let

$$
f=\sum_{\alpha \in \Pi} e_{-\alpha} \in \mathfrak{g} \simeq \mathfrak{g}^{*}
$$

(the last isomorphism is obtained from any non-degenerate inner product on $\mathfrak{g}$ which we fix once and for all) be the principal nilpotent element. Let $\{e, h, f\}$ be a principal $\mathfrak{s l}_{2}$-triple in $\mathfrak{g}$ containing $f$. The operator ad $h$ defines a gradation on $U(\mathfrak{g})$ which is called the principal gradation. The algebra $\mathcal{A}_{f}$ is generated by 
homogeneous elements with respect to the principal gradation on $U(\mathfrak{g})$. Moreover, the homomorphism $Z(\widehat{\mathfrak{g}}) \rightarrow \mathcal{A}_{f}$ is a homomorphism of graded algebras. The algebra $\mathcal{A}_{f}$ acts on $V_{\lambda}$ by creation operators.

The following result was proved in [3]:

THEOREM 2. The module $V_{\lambda}$ is cyclic as an $\mathcal{A}_{\mu}$-module for any regular $\mu \in \mathfrak{g}^{*}$. Moreover, if $\mu=f$, then the highest weight vector of $V_{\lambda}$ is a cyclic vector.

\subsection{THE ASSOCIATED GRADED OF $\operatorname{End}_{\widehat{\mathfrak{g}}}\left(\mathbb{V}_{\lambda}\right)$}

Each endomorphism of the Weyl module $\mathbb{V}_{\lambda}$ is uniquely determined by the image of the generating subspace $V_{\lambda} \subset \mathbb{V}_{\lambda}$. Hence the algebra $\operatorname{End}_{\widehat{\mathfrak{g}}}\left(\mathbb{V}_{\lambda}\right)$ may be naturally identified with

$$
\left(\operatorname{End}_{\mathbb{C}}\left(V_{\lambda}\right) \otimes U_{\kappa_{c}}(\widehat{\mathfrak{g}}) / \operatorname{End}_{\mathbb{C}}\left(V_{\lambda}\right) \otimes U_{\kappa_{c}}(\widehat{\mathfrak{g}})\left(\widehat{\mathfrak{g}}_{+}-\pi_{\lambda}\left(\widehat{\mathfrak{g}}_{+}\right)\right)\right)^{\widehat{\mathfrak{g}}_{+}} .
$$

Here $\operatorname{End}_{\mathbb{C}}\left(V_{\lambda}\right) \otimes U_{\kappa_{c}}(\widehat{\mathfrak{g}})\left(\widehat{\mathfrak{g}}_{+}-\pi_{\lambda}\left(\widehat{\mathfrak{g}}_{+}\right)\right)$is the left ideal generated by $x-\pi_{\lambda}(x)$ for all $x \in \widehat{\mathfrak{g}}_{+}$. Since each element of the above quotient algebra has a unique representative in $\operatorname{End}_{\mathbb{C}}\left(V_{\lambda}\right) \otimes U\left(\widehat{\mathfrak{g}}_{-}\right)$, the algebra $\operatorname{End}_{\widehat{\mathfrak{g}}}\left(\mathbb{V}_{\lambda}\right)$ may be regarded as a subalgebra $\mathcal{B} \subset \operatorname{End}_{\mathbb{C}}\left(V_{\lambda}\right) \otimes U\left(\widehat{\mathfrak{g}}_{-}\right)$(in the same way as in the previous subsection).

Let $\mathcal{Z} \subset \mathcal{B}=\operatorname{End}_{\widehat{\mathfrak{g}}}\left(\mathbb{V}_{\lambda}\right)$ be the image of the center of the completed enveloping algebra at the critical level $Z(\widehat{\mathfrak{g}}) \subset \widetilde{U}_{\kappa_{c}}(\widehat{\mathfrak{g}})$. Since each element of $\mathcal{B}$ commutes with $\widetilde{U}_{\kappa_{c}}(\widehat{\mathfrak{g}})$, we find that $\mathcal{Z}$ belongs to the center of $\mathcal{B}$.

Consider a filtration on the algebra $\operatorname{End}_{\mathbb{C}}\left(V_{\lambda}\right) \otimes U\left(\widehat{\mathfrak{g}}_{-}\right)$determined by the trivial filtration on the first factor and the PBW filtration on the second one. This filtration determines (by restriction to a subalgebra) filtrations on $\mathcal{B}$ and $\mathcal{Z}$. Due to the PBW theorem, the associated graded of $\operatorname{End}_{\mathbb{C}}\left(V_{\lambda}\right) \otimes U\left(\widehat{\mathfrak{g}}_{-}\right)$is

$$
\operatorname{End}_{\mathbb{C}}\left(V_{\lambda}\right) \otimes S\left(\widehat{\mathfrak{g}}_{-}\right) \simeq \operatorname{End}_{\mathbb{C}}\left(V_{\lambda}\right) \otimes \operatorname{Fun}(\mathfrak{g}[[t]]) .
$$

The isomorphism $S\left(\widehat{\mathfrak{g}}_{-}\right) \simeq$ Fun $(\mathfrak{g}[[t]])$ depends on the choice of an invariant nondegenerate scalar product $(\cdot, \cdot)$ on $\mathfrak{g}$. Namely, a linear element $x t^{-k} \in S\left(\widehat{\mathfrak{g}}_{-}\right)$maps to a linear function defined on $y(t) \in \mathfrak{g}[[t]]$ as $\operatorname{Res}_{t=0}(x, y(t)) t^{-k}$. In particular, $S\left(\mathfrak{g} t^{-1}\right)$ does to Fun $(\mathfrak{g})$ under this isomorphism.

The associated graded of $\mathcal{B}$,

$$
\overline{\mathcal{B}}:=\operatorname{gr} \mathcal{B}=\operatorname{gr}\left(\operatorname{End}_{\mathbb{C}}\left(V_{\lambda}\right) \otimes U_{\kappa_{c}}(\widehat{\mathfrak{g}}) / \operatorname{End}_{\mathbb{C}}\left(V_{\lambda}\right) \otimes U_{\kappa_{c}}(\widehat{\mathfrak{g}})\left(\widehat{\mathfrak{g}}_{+}-\pi_{\lambda}\left(\widehat{\mathfrak{g}}_{+}\right)\right)\right)^{\widehat{\mathfrak{g}}_{+}},
$$

is naturally embedded into

$$
\begin{aligned}
& \left(\operatorname{gr}\left(\operatorname{End}_{\mathbb{C}}\left(V_{\lambda}\right) \otimes U_{\kappa_{c}}(\widehat{\mathfrak{g}}) / \operatorname{End}_{\mathbb{C}}\left(V_{\lambda}\right) \otimes U_{\kappa_{c}}(\widehat{\mathfrak{g}})\left(\widehat{\mathfrak{g}}_{+}-\pi_{\lambda}\left(\widehat{\mathfrak{g}}_{+}\right)\right)\right)\right)^{\widehat{\mathfrak{g}}_{+}} \simeq \\
& \quad \simeq\left(\operatorname{End}_{\mathbb{C}}\left(V_{\lambda}\right) \otimes \operatorname{Fun}(\mathfrak{g}[[t]])\right)^{\mathfrak{g}[[t]]} .
\end{aligned}
$$

However, it was shown in [9] that this embedding is not an isomorphism unless $\lambda=0$ or minuscule (the reason for this is that it is only for these $\lambda$ that the 
module $V_{\lambda}$ is cyclic for the centralizer $\mathfrak{a}_{f}$ of the principal nilpotent element $f$ ). Nevertheless, we will now use our results on the shift of argument subalgebra from [3] to give an estimate of the image of $\overline{\mathcal{B}}$ in (5), which will turn out to be sufficient for our purposes.

The subalgebra

$$
\overline{\mathcal{Z}}:=\operatorname{gr} \mathcal{Z} \subset\left(\operatorname{End}_{\mathbb{C}}\left(V_{\lambda}\right) \otimes \operatorname{Fun}(\mathfrak{g}[[t]])\right)^{\mathfrak{g}[[t]]}
$$

is generated by the elements $\pi_{\lambda} \otimes 1\left(\overline{P_{i}^{(k)}}\right)$ with $i=1, \ldots, \mathrm{rkg}, k \geq 0$ (this follows from the definition of the elements $\left.\overline{P_{i}^{(k)}}\right)$.

Consider the subalgebra

$$
\overline{\mathcal{Z}^{\prime}}:=1 \otimes \operatorname{Fun}(\mathfrak{g}[[t]])^{\mathfrak{g}[[t]]} \subset\left(\operatorname{End}_{\mathbb{C}}\left(V_{\lambda}\right) \otimes \operatorname{Fun}(\mathfrak{g}[[t]])\right)^{\mathfrak{g}[[t]]} .
$$

LEMMA 2. The algebra $\overline{\mathcal{Z}^{\prime}}$ is a free polynomial algebra generated by $\pi_{\lambda} \otimes 1\left(\overline{P_{i}^{(k)}}\right)$ with $i=1, \ldots, \mathrm{rk} \mathfrak{g}, k \geq d_{i}+1$, and hence $\overline{\mathcal{Z}^{\prime}} \subset \overline{\mathcal{Z}}$.

Proof. According to a result of [1] (see [9, Theorem 3.4.2], for a proof), the algebra $\overline{\mathcal{Z}^{\prime}}$ is a free polynomial algebra generated by some homogeneous elements $\overline{S_{i}^{(k)}}$ with $i=1, \ldots, \mathrm{rk} \mathfrak{g}, k \geq d_{i}+1$ of degrees $k$ with respect to the loop-rotation grading and $d_{i}+1$ with respect to the grading by the degree of polynomials. Hence the elements $\overline{P_{i}^{(k)}}$ with $i=1, \ldots$, rk $\mathfrak{g}, k \geq d_{i}+1$ generate a free polynomial subalgebra of the same size in $(U(\mathfrak{g}) \otimes \operatorname{Fun}(\mathfrak{g}[[t]]))^{\mathfrak{g}[[t]]}$. Thus it remains to show that $\overline{P_{i}^{(k)}} \in$ $1 \otimes \operatorname{Fun}(\mathfrak{g}[[t]])^{\mathfrak{g}[[t]]}$. Note that the elements $P_{i}^{(k)} \in \mathcal{Z}$ with $i=1, \ldots$, rk $\mathfrak{g}, k \geq d_{i}+1$ are homogeneous with respect to the loop-rotation grading, and hence their leading terms with respect to the PBW filtration belong to $1 \otimes U\left(\widehat{\mathfrak{g}}_{-}\right)$. Therefore $\overline{P_{i}^{(k)}} \in$ $1 \otimes \operatorname{Fun}(\mathfrak{g}[[t]])^{\mathfrak{g}[[t]]}$. Hence the assertion.

Let $\mathcal{I}$ be the left ideal in $\left(\operatorname{End}_{\mathbb{C}}\left(V_{\lambda}\right) \otimes \operatorname{Fun}(\mathfrak{g}[[t]])\right)^{\mathfrak{g}[t]]]}$ generated by all $\overline{P_{i}^{(k)}}$ $\left(i=1, \ldots, \mathrm{rkg}, k \geq d_{i}+1\right)$. According to [9], Sects. 9.6.4-9.6.5, the algebra $\left(\operatorname{End}_{\mathbb{C}}\left(V_{\lambda}\right) \otimes \operatorname{Fun}(\mathfrak{g}[[t]])\right)^{\mathfrak{g}[[t]]}$ is a free $\overline{\mathcal{Z}^{\prime}}$-module. Any space of generators of this module is therefore isomorphic to the space of $\mathcal{I}$-coinvariants

$$
\left(\operatorname{End}_{\mathbb{C}}\left(V_{\lambda}\right) \otimes \operatorname{Fun}(\mathfrak{g}[[t]])\right)^{\mathfrak{g}[t]]} / \mathcal{I} \simeq\left(\operatorname{End}_{\mathbb{C}}\left(V_{\lambda}\right) \otimes \operatorname{Fun}(\mathfrak{g})\right)^{\mathfrak{g}} / 1 \otimes \operatorname{Fun}(\mathfrak{g})_{+}^{\mathfrak{g}},
$$

where $\operatorname{Fun}(\mathfrak{g})_{+}^{\mathfrak{g}}$ is the maximal graded ideal in $\operatorname{Fun}(\mathfrak{g})^{\mathfrak{g}}$.

According to [10], the latter quotient has the following description. Let $f \in \mathfrak{g}=$ $\mathfrak{g} \cdot 1 \subset \mathfrak{g}[[t]]$ be the principal nilpotent element (4). The evaluation homomorphism at $f$,

$\operatorname{id} \otimes \operatorname{ev}_{f}: \operatorname{End}_{\mathbb{C}}\left(V_{\lambda}\right) \otimes \operatorname{Fun}(\mathfrak{g}[[t]]) \rightarrow \operatorname{End}_{\mathbb{C}}\left(V_{\lambda}\right)$ 
annihilates $\mathcal{I}$ and gives rise to an isomorphism

$$
\left(\operatorname{End}_{\mathbb{C}}\left(V_{\lambda}\right) \otimes \operatorname{Fun}(\mathfrak{g}[[t]])\right)^{\mathfrak{g}[[t]]} / \mathcal{I} \simeq \operatorname{End}_{\mathfrak{a}_{f}}\left(V_{\lambda}\right),
$$

where $\mathfrak{a}_{f} \subset \mathfrak{g}$ is the centralizer of $f$. This is an isomorphism of graded algebras with respect to the loop-rotation grading (defined by the operator $-t \partial_{t}$ ) on the left-hand side and the principal grading on the right-hand side.

Let now $\mathcal{A}_{f} \subset U(\mathfrak{g})$ be the quantum shift of argument subalgebra corresponding to the principal nilpotent element $f \in \mathfrak{g}=\mathfrak{g}^{*}$.

LEMMA 3. There is a commutative diagram of graded algebras (with respect to the loop-rotation grading on the left-hand side and the principal grading on the righthand side).

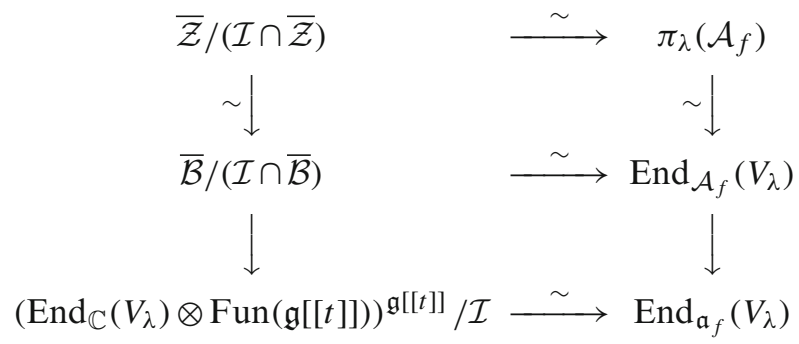

Proof. By definition of the quantum shift of argument subalgebra, id $\otimes f_{-}(\mathcal{Z})$ is $\pi_{\lambda}\left(\mathcal{A}_{f}\right) \subset \operatorname{End}_{\mathbb{C}}\left(V_{\lambda}\right)$. Since $\operatorname{gr} f_{-}=\operatorname{ev}_{f}$, Lemma 1 implies that $\operatorname{id} \otimes \operatorname{ev}_{f}(\overline{\mathcal{Z}})=$ $\pi_{\lambda}\left(\mathcal{A}_{f}\right)$. Since $\mathcal{Z}$ belongs to the center of $\mathcal{B}$, we have id $\otimes \mathrm{ev}_{f}(\overline{\mathcal{B}}) \subset \operatorname{End}_{\mathcal{A}_{f}}\left(V_{\lambda}\right)$.

On the other hand, according to Theorem 2, the algebra $\mathcal{A}_{f}$ has a cyclic vector in $V_{\lambda}$. Hence

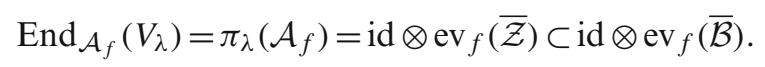

Thus, we obtain the opposite inclusion, which proves the assertion of the lemma.

Now we are going to prove that $\overline{\mathcal{B}}=\overline{\mathcal{Z}}$. Note that the algebra $\overline{\mathcal{Z}}$ is generated over $\overline{\mathcal{Z}}^{\prime}$ by the elements $\pi_{\lambda} \otimes 1\left(\overline{P_{i}^{(k)}}\right)$ with $i=1, \ldots$, rk $\mathfrak{g}, k \leq d_{i}$. Due to the same "homogeneity" argument as in Lemmas 1 and 2 we find that $\pi_{\lambda} \otimes 1\left(\overline{P_{i}^{(k)}}\right)$ with $i=1, \ldots, \mathrm{rk} \mathfrak{g}, \quad k \leq d_{i}$ belong to $\operatorname{End}_{\mathbb{C}}\left(V_{\lambda}\right) \otimes S\left(\mathfrak{g} t^{-1}\right)=\operatorname{End}_{\mathbb{C}}\left(V_{\lambda}\right) \otimes \operatorname{Fun}(\mathfrak{g})$. Hence we can lift the graded space $\overline{\mathcal{Z}} /(\mathcal{I} \cap \overline{\mathcal{Z}})=\overline{\mathcal{B}} /(\mathcal{I} \cap \overline{\mathcal{B}})$ to a graded subspace $N \subset$ $\overline{\mathcal{Z}} \cap \operatorname{Fun}(\mathfrak{g})$.

LEMMA 4. $\overline{\mathcal{B}}$ and $\overline{\mathcal{Z}}$ are both free $\overline{\mathcal{Z}}^{\prime}$-modules generated by $N$.

Proof. Let $M \subset\left(\operatorname{End}_{\mathbb{C}}\left(V_{\lambda}\right) \otimes \operatorname{Fun}(\mathfrak{g})\right)^{\mathfrak{g}}$ be a space of generators of $\left(\operatorname{End}_{\mathbb{C}}\left(V_{\lambda}\right) \otimes \operatorname{Fun}(\mathfrak{g}[[t]])\right)^{\mathfrak{g}[[t]]}$ 
as a free $\overline{\mathcal{Z}^{\prime}}$-module, containing $N$. Clearly, $M$ also freely generates the $\operatorname{Fun}(\mathfrak{g})^{\mathfrak{g}_{-}}$ $\operatorname{module}\left(\operatorname{End}_{\mathbb{C}}\left(V_{\lambda}\right) \otimes \operatorname{Fun}(\mathfrak{g})\right)^{\mathfrak{g}}$.

For each regular $\mu_{0} \in \mathfrak{g}$, the evaluation at $\mu_{0}$ gives us an isomorphism of vector spaces

$$
\operatorname{id} \otimes \operatorname{ev}_{\mu_{0}}: M \stackrel{\sim}{\longrightarrow} \operatorname{End}_{\mathfrak{z} \mathfrak{g}\left(\mu_{0}\right)} V_{\lambda},
$$

where $\mathfrak{z}_{\mathfrak{g}}\left(\mu_{0}\right)$ is the centralizer of $\mu_{0}$ in $\mathfrak{g}$ (this is a classical result due to Kostant [10]). Since $N \subset \overline{\mathcal{Z}}$, we have $\operatorname{id} \otimes \operatorname{ev}_{\mu_{0}}(N) \subset \pi_{\lambda}\left(\mathcal{A}_{\mu_{0}}\right)$. Since $\operatorname{id} \otimes \operatorname{ev}_{\mu_{0}}$ is an injection on $M$, we have $\operatorname{dim}\left(\operatorname{id} \otimes \operatorname{ev}_{\mu_{0}}(N)\right)=\operatorname{dim} N=\operatorname{dim} V_{\lambda}=\operatorname{dim} \pi_{\lambda}\left(\mathcal{A}_{\mu_{0}}\right)$. Hence, for each regular $\mu_{0} \in \mathfrak{g}$, the evaluation at $\mu_{0}$ gives us an isomorphism of vector spaces

$$
\operatorname{id} \otimes \operatorname{ev}_{\mu_{0}}: N \stackrel{\sim}{\longrightarrow} \pi_{\lambda}\left(\mathcal{A}_{\mu_{0}}\right) .
$$

Therefore, for any $\mu=\mu_{0}+t \mu_{1}+\cdots \in \mathfrak{g}[[t]]$, we have $\operatorname{id} \otimes \operatorname{ev}_{\mu}\left(\overline{\mathcal{Z}}^{\prime} \cdot N\right)=\pi_{\lambda}\left(\mathcal{A}_{\mu_{0}}\right)$.

The submodules $\overline{\mathcal{B}} \subset \overline{\mathcal{Z}}^{\prime} \cdot M$ and $\overline{\mathcal{Z}} \subset \overline{\mathcal{Z}}^{\prime} \cdot M$ clearly contain $\overline{\mathcal{Z}}^{\prime} \cdot N$. Since $\overline{\mathcal{Z}}^{\prime} \cdot N \subset$ $\overline{\mathcal{Z}}$, the subalgebras $\operatorname{id} \otimes \operatorname{ev}_{\mu}(\overline{\mathcal{Z}}) \subset \operatorname{End}_{\mathbb{C}} V_{\lambda}$ and $\operatorname{ev}_{\mu}(\overline{\mathcal{B}}) \subset \operatorname{End}_{\mathbb{C}} V_{\lambda}$ belong to the commutant of id $\otimes \operatorname{ev}_{\mu}(N)=\pi_{\lambda}\left(\mathcal{A}_{\mu_{0}}\right)$ in $\operatorname{End}_{\mathbb{C}} V_{\lambda}$ for any $\mu=\mu_{0}+t \mu_{1}+\cdots \in \mathfrak{g}[[t]]$ with regular $\mu_{0}$. By Theorem 2, $V_{\lambda}$ is a cyclic $\mathcal{A}_{\mu_{0}}$-module and hence $\pi_{\lambda}\left(\mathcal{A}_{\mu_{0}}\right)=$ End $_{\mathcal{A}_{\mu_{0}}}\left(V_{\lambda}\right)$. Thus we have

$$
\operatorname{id} \otimes \operatorname{ev}_{\mu}\left(\overline{\mathcal{Z}}^{\prime} \cdot N\right)=\pi_{\lambda}\left(\mathcal{A}_{\mu_{0}}\right)=\operatorname{End}_{\mathcal{A}_{\mu_{0}}}\left(V_{\lambda}\right)=\operatorname{id} \otimes \operatorname{ev}_{\mu}(\overline{\mathcal{B}})=\operatorname{id}_{\operatorname{ev}_{\mu}}(\overline{\mathcal{Z}}) .
$$

Since $\overline{\mathcal{Z}}^{\prime}$ is the algebra of $\mathfrak{g}[[t]]$-invariant functions on $\mathfrak{g}[[t]]$, each point of $\operatorname{Spec} \overline{\mathcal{Z}}^{\prime}$ has a representative in $\mathfrak{g}[[t]]$ of the form $\mu=\mu_{0}+t \mu_{1}+\cdots \in \mathfrak{g}[[t]]$ with regular $\mu_{0}$. Therefore we find that the images of $\overline{\mathcal{B}}$ and $\overline{\mathcal{Z}}$ in the quotient of $\overline{\mathcal{Z}}^{\prime} \cdot M$ by each maximal ideal of $\overline{\mathcal{Z}}^{\prime}$ have the same dimension equal to $\operatorname{dim} N=\operatorname{dim} V_{\lambda}$.

Let $C$ be a complementary subspace to $N$ in the space of generators $M$, and let $c_{1}, \ldots, c_{n}$ be a basis of $C$. Then $\overline{\mathcal{Z}}^{\prime} \cdot M=\overline{\mathcal{Z}}^{\prime} \cdot N \oplus \overline{\mathcal{Z}}^{\prime} \cdot C$. Suppose that $\overline{\mathcal{B}} \neq \overline{\mathcal{Z}}^{\prime} \cdot N$. Then the module $\overline{\mathcal{B}}$ contains an element from $\overline{\mathcal{Z}}^{\prime} \cdot C$ of the form $z_{1} c_{1}+\cdots+z_{n} c_{n}$ with $z_{i} \in \overline{\mathcal{Z}}^{\prime}$. Choose a maximal ideal $J \subset \overline{\mathcal{Z}}^{\prime}$ such that $z_{1} \notin J$ (i.e. $\mu=\mu_{0}+t \mu_{1}+$ $\cdots \in \mathfrak{g}[[t]]$ with regular $\mu_{0}$ such that $\left.z_{1}(\mu) \neq 0\right)$. Then $\overline{\mathcal{B}} /(\overline{\mathcal{B}} \cap J \cdot M) \supset N+c_{1}$ and therefore $\operatorname{dim} \overline{\mathcal{B}} /(\overline{\mathcal{B}} \cap J \cdot M)>\operatorname{dim} N$. On the other hand, we have found above that $\operatorname{dim} \overline{\mathcal{B}} /(\overline{\mathcal{B}} \cap J \cdot M)=\operatorname{dim} N$ for all maximal ideals $J$. Hence we obtain a contradiction. In the same way we prove that $\overline{\mathcal{Z}}=\overline{\mathcal{Z}} \cdot N$.

COROLLARY $1 . \mathcal{B}=\mathcal{Z}$.

Thus, the homomorphism $Z(\widehat{\mathfrak{g}}) \rightarrow \operatorname{End}_{\widehat{\mathfrak{g}}}\left(\mathbb{V}_{\lambda}\right)$ is surjective. Hence it remains to show that the character of $\mathcal{Z}$ (or, equivalently, $\overline{\mathcal{Z}}$ ) is not smaller than that of $\operatorname{Fun}\left(\mathrm{Op}_{L_{G}}^{\lambda, \text { reg }}\right)$. This will be done in the next section. 


\subsection{COMPARISON OF CHARACTERS}

According to [3], the character of $\pi_{\lambda}\left(\mathcal{A}_{f}\right)$ is the same as that of $V_{\lambda}$ with respect to the principal grading, which is known to be

$$
\frac{\prod_{\alpha>0}\left(1-q^{\left\langle\alpha^{\vee}, \lambda+\rho\right\rangle}\right)}{\prod_{\alpha>0}\left(1-q^{\left\langle\alpha^{\vee}, \rho\right\rangle}\right)}
$$

The denominator may be rewritten as

$$
\prod_{\alpha>0}\left(1-q^{\left\langle\alpha^{\vee}, \rho\right\rangle}\right)=\prod_{i=1}^{\mathrm{rkg} g} \prod_{k=1}^{d_{i}}\left(1-q^{k}\right) .
$$

On the other hand, the character of $\overline{\mathcal{Z}^{\prime}}$ (with respect to the loop-rotation grading) is $\prod_{i=1}^{\mathrm{rkg}} \prod_{k=d_{i}+1}^{\infty}\left(1-q^{k}\right)$. According to Lemmas 3 and $4, \overline{\mathcal{Z}}$ is a free $\overline{\mathcal{Z}}^{\prime}$-module with the space of generators $\pi_{\lambda}\left(\mathcal{A}_{f}\right)$. Hence the character of $\overline{\mathcal{Z}}$ is the product of those of $\pi_{\lambda}\left(\mathcal{A}_{f}\right)$ and $\overline{\mathcal{Z}}^{\prime}$. Therefore it is equal to

$$
\frac{\prod_{\alpha>0}\left(1-q^{\left\langle\alpha^{\vee}, \lambda+\rho\right\rangle}\right)}{\prod_{k=1}^{\infty}\left(1-q^{k}\right)^{\mathrm{rk} \mathfrak{g}}}
$$

which coincides with the character of $\operatorname{Fun}\left(\mathrm{Op}_{L_{G}}^{\lambda, \text { reg }}\right)$ given by formula (3).

Since $\mathcal{Z}$ factors through $\operatorname{Fun}\left(\mathrm{Op}_{L_{G}}^{\lambda, \text { reg }}\right)$ by Proposition 1 and we have shown that the homomorphism $Z(\widehat{\mathfrak{g}}) \rightarrow \operatorname{End}_{\widehat{\mathfrak{g}}}\left(\mathbb{V}_{\lambda}\right)$ is surjective, this completes the proof of Theorem 1.

\section{Acknowledgements}

We thank D. Gaitsgory for useful comments on the draft of this paper.

Open Access This article is distributed under the terms of the Creative Commons Attribution Noncommercial License which permits any noncommercial use, distribution, and reproduction in any medium, provided the original author(s) and source are credited.

\section{References}

1. Beilinson, A., Drinfeld, V.: Quantization of Hitchin's integrable system and Hecke eigensheaves (preprint). Available at http://www.ma.utexas.edu/ benzvi/BD

2. Feigin, B., Frenkel, E.: Affine Kac-Moody algebras at the critical level and GelfandDikii algebras. Int. J. Mod. Phys. A7(Suppl 1A), 197-215 (1992)

3. Feigin, B., Frenkel, E., Rybnikov, L.: Opers with irregular singularity and spectra of the shift of argument subalgebra (preprint) (math.QA/0712.1183)

4. Feigin, B., Frenkel, E., Toledano Laredo, V.: Gaudin model with irregular singularities (preprint) (math.QA/0612798)

5. Frenkel, E., Gaitsgory, D.: Weyl modules and opers without monodromy (preprint) (math.QA/0706.3725) 
6. Frenkel, E., Gaitsgory, D.: Local geometric Langlands correspondence and affine Kac-Moody algebras. Algebraic Geometry and Number Theory, Progress in Math., vol. 253, pp. 69-260. Birkhäuser Boston (2006) (math.RT/0508382)

7. Frenkel, E., Gaitsgory, D.: Fusion and convolution: applications to affine Kac-Moody algebras at the critical level. Pure Appl. Math. Quart. 2, 1255-1312 (2006) (math.RT/0511284)

8. Frenkel, E.: Wakimoto modules, opers and the center at the critical level. Adv. Math. 195, 297-404 (2005) (math.QA/0210029)

9. Frenkel, E.: Langlands correspondence for loop groups. Cambridge Studies in Advanced Mathematics, vol. 103. Cambridge University Press, Cambridge (2007)

10. Kostant, B.: Lie group representations on polynomial rings. Am. J. Math. 85, 327404 (1963)

11. Rybnikov, L.: Argument shift method and Gaudin model. Func. Anal. Appl. 40(3) (2006) [translated from Funktsional'nyi Analiz i Ego Prilozheniya 40(3), 30-43 (2006) (math.RT/0606380)] 